\title{
Analysis Based on RBF Neural Network for Effect of Artificial Flow Field on Dissolved Oxygen Uniform Distribution
}

\author{
Gang LIU \\ College of Electrical \\ Guangxi University \\ Nanning, Guangxi, China \\ E-mail: liugang5000@163.com \\ Zengqiang LEI \\ College of Electrical \\ Guangxi University \\ Nanning, Guangxi, China \\ E-mail: 604723614@qq.com
}

\author{
Jiaoyan $\mathrm{AI}^{*}$ \\ College of Electrical \\ Guangxi University \\ Nanning, Guangxi, China \\ E-mail: shinin@vip.163.com \\ +*Corresponding author \\ Kui LIANG \\ College of Electrical \\ Guangxi University \\ Nanning, Guangxi, China \\ E-mail: 1216231989@qq.com
}

\begin{abstract}
To verify the effect of artificial flow on the improvement of local DO(dissolved oxygen) imbalance distribution, Lake Jing in Guangxi University was taken as the experimental area, five groups of experiments were designed, pump with different flow rate and different nozzle were used to push flow in experimental area, RBF Neural Network which is widely used in geographic information prediction was used to fitting the spatial distribution of dissolved oxygen and the dispersion coefficient was taken as the equilibrium index of DO horizontal distribution to analysis the effect of flow. The result shows that100 $\mathrm{m}^{3} / \mathrm{min}, 130 \mathrm{~m}^{3} / \mathrm{min}, 180 \mathrm{~m}^{3} / \mathrm{min}$ pump with circular nozzle and $130 \mathrm{~L} / \mathrm{min}$ pump with duckbill nozzle respectively need 1.5 hour, 1.0 hour, 0.5 hour, 1.5 hour to decrease the DO dispersion coefficient from 0.3 to 0.05 , witch prove push flow has obvious effect on improving the DO balance and the effect is related with flow rate and nozzle, a larger flow pump and circular nozzle is preferred.
\end{abstract}

Keywords-artificial flow field; dissolved oxygen; uniform distribution; RBF neural network

\section{INTRODUCTION}

Affected by natural factors like light, grass growth etc. the distribution of dissolved oxygen in artificial landscape water and aquaculture water present a large difference [1], mainly reflects in oxygen concentration is too low or too high in some local water. if the concentration is too low, survival of the fish and other biological will be threatened, and anaerobic bacteria reproduction will be promoted, causing the deterioration of the water quality in this area, so that affect whole the water body; While the high concentration causing fish aggregation so that reduce fish production per unit area.

So evenly distribution of Dissolved oxygen is necessary. For water flow has great influence on the dissolved oxygen distribution [2], we propose to improve the dissolved oxygen distribution by artificial water flow. In order to verify the effect of improvement of different flow field, several sets experiments were designed, we use pumps with different flow and different nozzle to produce different flow field in experimental area, sampling the DO with equal interval by time, and use RBF neural network to reconstruction the DO distribution in experimental area. The result proves that flow filed has obvious improvement effect on uniform distribution of DO.

\section{EXPERIMENTAL DESIGN}

\section{A. Experimental Area}

Lake Jing located in Guangxi University, for uneven of shade cover and growth of plants, the DO distribution present large difference, we chose a $7 \mathrm{~m} * 5 \mathrm{~m}$ area located in east coast side as experimental area shown in Fig. 1.

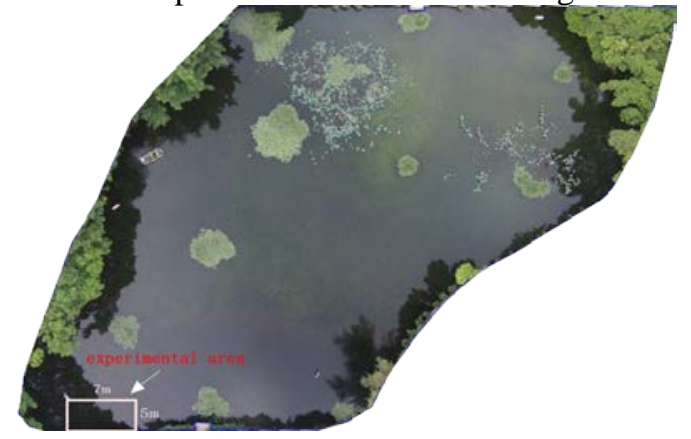

Figure 1. Experimental area

\section{B. Experimental Grouping}

Five groups experiment were designed as shown in Table I, we place pumps of $100 \mathrm{~m}^{3} / \mathrm{min}, 130 \mathrm{~m}^{3} / \mathrm{min}, 180 \mathrm{~m}^{3} / \mathrm{min}$ with circular nozzle in center of the shore five centimeters underwater to produce vertical flow field, a group of 130 $\mathrm{m}^{3} / \mathrm{min}$ with duckbill nozzle and a group with no pump were designed as comparison. 
Experiments were performed at 8:30-11:00 every day, and sampled the DO at equal intervals in experimental area as shown in Fig. 2, the experimental area were divided into 35 meshes at intervals of $1 \mathrm{~m}^{*} 1 \mathrm{~m}$, all grids were sampled each time.

TABLE I. EXPERIMENTAL GROUPING

\begin{tabular}{|c|c|c|c|}
\hline \multirow{2}{*}{ Group } & \multicolumn{3}{|c|}{ Parameter } \\
\cline { 2 - 4 } & Pump power & Pump flow & Nozzle shape \\
\hline 1 & $120 \mathrm{~W}$ & $100 \mathrm{~m}^{3} / \mathrm{min}$ & circular \\
\hline 2 & $130 \mathrm{~W}$ & $130 \mathrm{~m}^{3} / \mathrm{min}$ & circular \\
\hline 3 & $180 \mathrm{~W}$ & $180 \mathrm{~m}^{3} / \mathrm{min}$ & circular \\
\hline 4 & $130 \mathrm{~W}$ & $130 \mathrm{~m}^{3} / \mathrm{min}$ & duckbill \\
\hline 5 & \multicolumn{3}{|c|}{ No pump } \\
\hline
\end{tabular}

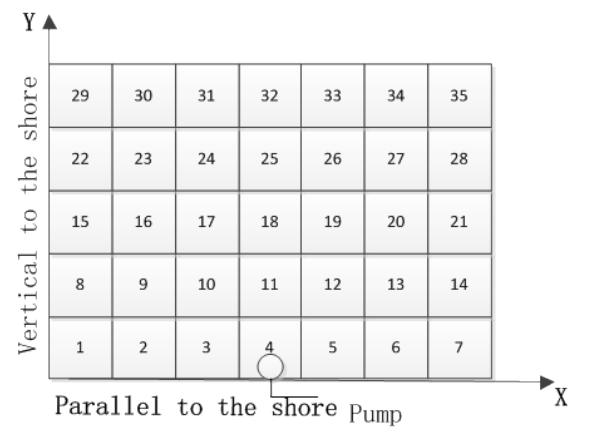

Figure 2. RBF network structure

\section{RBF Based Spatial Fitting of Dissolved OXYGEN DISTRIBUTION}

Experimental data for each measurement is only the dissolved oxygen concentration value at the center of discrete grid, it is not reasonable to regard that DO concentration at each position in one grid are equals, so the sparse discrete sampled data cannot reflect the DO distribution on whole experimental area precisely and effectively. Therefore to obtain more dense data to reflect the distribution of $\mathrm{DO}$ in the experimental area more accurately, it is required to perform spatial interpolation at experimental area [3-4].

Spatial interpolation use the coordinates and DO concentration of known points to fit the relationship between the coordinates and DO concentration to speculate the DO concentration at the unknown points that is to obtain the relationship $\mathrm{z}=\mathrm{f}(\mathrm{x}, \mathrm{y})$, here $\mathrm{x}, \mathrm{y}$ represent coordinate and $\mathrm{z}$ represents the corresponding DO concentration.

In this paper we use RBF neural network to fit the relationship between coordinate and DO concentration, RBF can approach any nonlinear function, which has good generalization ability and has a fast convergence rate, which is widely used in geographic information prediction [5-6]. The network structure is shown as Fig. 3, the coordinates of sampling point were taken as input and corresponding DO concentration were taken as output [7].

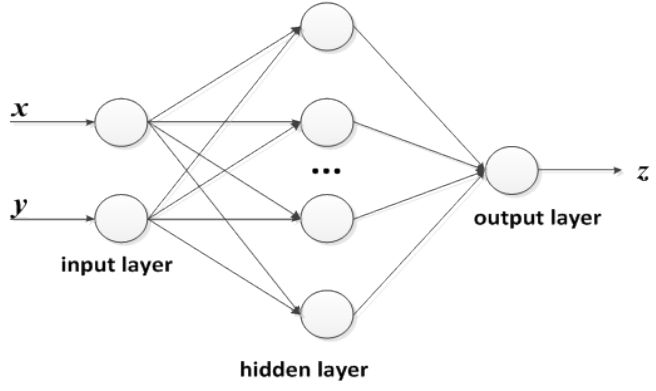

Figure 3. RBF network structure

The fitting step as follows:

The spatial distribution of dissolved oxygen fitting is performed at MATLAB neural network toolbox [8].

- Data normalization: normalized the sampling point coordinates ( $\mathrm{x}, \mathrm{y})$ and the dissolved oxygen concentration according to the formula (1),(2),(3).

$$
\begin{aligned}
& x=\left(x-x_{\min }\right) /\left(x_{\max }-x_{\min }\right) \\
& y=\left(y-y_{\min }\right) /\left(y_{\max }-y_{\min }\right) \\
& Z=\left(Z-Z_{\min }\right) /\left(Z_{\text {max }}-Z_{\text {min }}\right)
\end{aligned}
$$

- Network training: divide the 35 sampled data into training set and test set according to the ratio of 7:3, create radial basis function neural network by using MATLAB neural network toolbox and substitute the training samples, the command as follows: net $=$ newrb $(P, T$, goal,spread $, m n, d f)$ net-neural network model; newrb-MATLAB command; $P$-input matrix, coordinates;

$T$-output matrix,DO concentration; goal-mse function; spread-Expansion factor;

$m n-$ Maximum number of hidden neurons; $d f$-Frequency of iteration.

- Substitute the test sets samples into the trained network to obtain the predictive value and compare with actual sampled data. "Trial and error method" was used to determine the spread coefficient spread and maximum number of hidden layer neurons $\mathrm{mn}$.

- Network simulation: Using the meshgrid command to generate the $400 * 600$ coordinates interpolation point in the experimental area, substitute the coordinates as the input of the trained RBF network after normalization, and use the same formula to anti-normalize the result, at last we can obtain the dissolved oxygen concentration value corresponding to the interpolation point. 


\section{IMPROVEMENT OF DISSOLVED OXYGEN DISTRIBUTION}

\section{A. Index of Uniform Distribution}

To compare DO distribution at different time, a index of uniform distribution is necessary. For the large different average value DO concentration at different time, we chose dispersion coefficient $\mathrm{Vx}$ as the index to measure the uniformity of DO concentration[9-10], the compute formula as follows:

$$
\begin{gathered}
V x=\frac{\sqrt{\frac{1}{n} \sum_{i=1}^{n}\left(x_{i}-\bar{x}\right)^{2}}}{\bar{x}} \\
x_{i}-\text { DO concentration at each interpolation point; } \\
\frac{x}{x} \text { - Average of DO concentration; } \\
n-\text { Number of interpolation points. }
\end{gathered}
$$

\section{B. Variation Trend of DO Dispersion Coefficient}

Calculate the DO dispersion coefficient at each time by formula (4) after performing spatial interpolation by RBF, and the part of the result as shown in Table II, group number in the table are correspond with Table I, time represents each sampling time. As the result shows that at 8:30, for each experiment performed at a similar weather, so the initial DO dispersion coefficient are similar, all distribute in the vicinity of 0.3 ; At 11:00, group 1-4 performed push flow, and the dispersion coefficient are about 0.05; Group 5 has not performed push flow, and it's dispersion coefficient are about 0.2; These shows that termination DO dispersion coefficient with push flow is significantly lower than case of none push flow.

TABLE II. EXPERIMENTAL RESULTS

\begin{tabular}{|c|c|c|c|c|c|c|}
\hline Group & $8: 30$ & $9: 00$ & $9: 30$ & $10: 00$ & $10: 30$ & $11: 00$ \\
\hline 1 & 0.341 & 0.192 & 0.090 & 0.066 & 0.033 & 0.034 \\
\hline 1 & 0.318 & 0.186 & 0.099 & 0.065 & 0.049 & 0.034 \\
\hline 2 & 0.349 & 0.158 & 0.069 & 0.052 & 0.031 & 0.037 \\
\hline 2 & 0.344 & 0.178 & 0.044 & 0.040 & 0.053 & 0.053 \\
\hline 3 & 0.294 & 0.064 & 0.055 & 0.040 & 0.049 & 0.053 \\
\hline 3 & 0.269 & 0.061 & 0.048 & 0.041 & 0.052 & 0.040 \\
\hline 4 & 0.292 & 0.175 & 0.113 & 0.072 & 0.043 & 0.034 \\
\hline 4 & 0.342 & 0.209 & 0.132 & 0.064 & 0.032 & 0.035 \\
\hline 5 & 0.303 & 0.275 & 0.263 & 0.238 & 0.215 & 0.212 \\
\hline 5 & 0.278 & 0.269 & 0.242 & 0.198 & 0.182 & 0.185 \\
\hline \multicolumn{7}{|c|}{} \\
\hline
\end{tabular}

Select an experiment from group 1-4(performed push flow) and an experiment from group 5(no flow), draw the interpolation result at 8:30 and 11:00 respectively into pseudo color picture and histogram as Fig. 4 and Fig. 5, block area in the figure represents the experimental area, DO concentration is represented by color, the corresponding relation of color and DO concentration as the color bar right side. At 8:30, both DO concentration presents a trapezoidal distribution and distribution of histogram are wide, which means the initial distribution of DO concentration is extremely non-uniform; At 11:00, the DO distribution of group 1-4 is no longer presents an obvious trapezoidal distribution, and it's histogram also become concentrated which means it's DO distribution is improved while the group 5 is still shows a trapezoidal distribution pseudo color picture and wide distribution histogram. By above comparison, it shows that push-flow has obvious effect on the improvement of DO distribution.
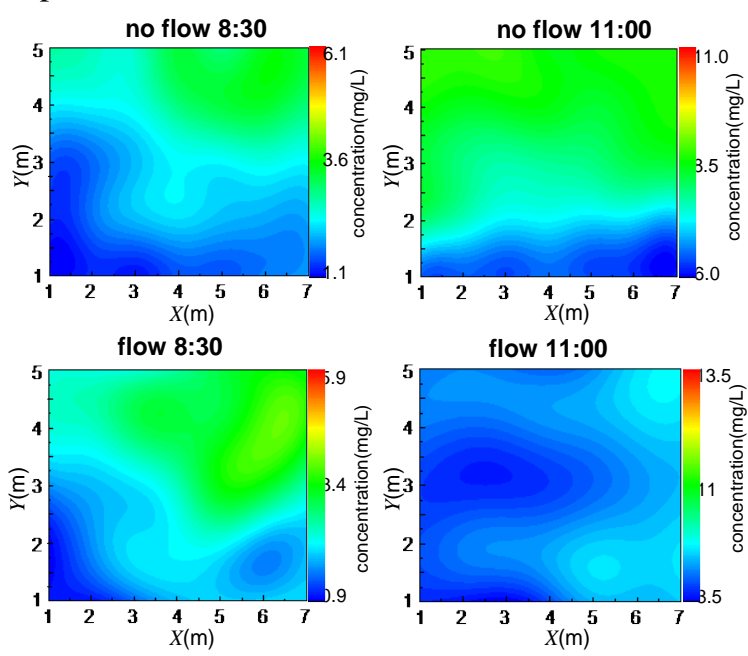

Figure 4. DO Distribution Pseudo-color pic
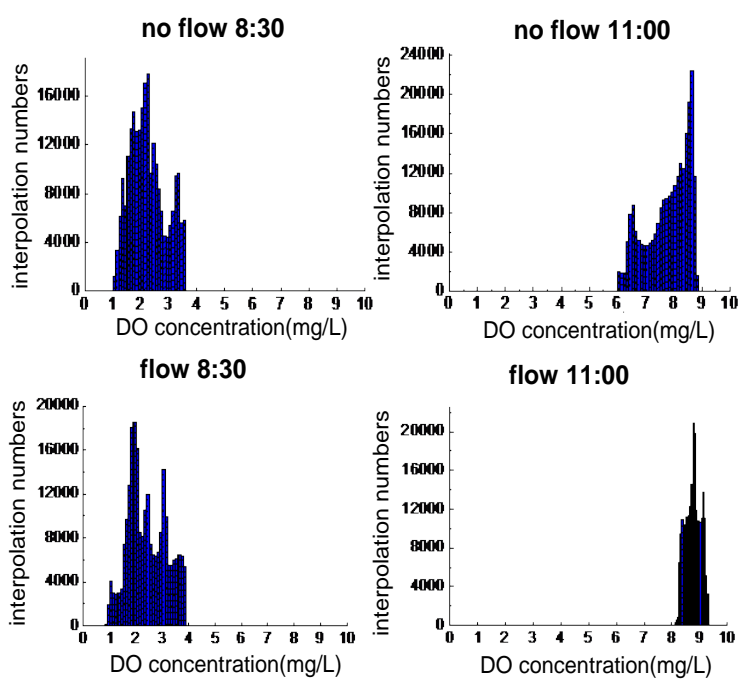

Figure 5. DO Distribution Histogram

The Fig. 6 is the decline curve of DO dispersion coefficient of group 1,2,3,5 (The difference is pump flow), the figure shows that in the case of push-flow (group 1, 2, 3), DO dispersion coefficient has a significant decline over time and finally stabilized at 0.05 , while in the case of no push flow (group 5), DO dispersion coefficient changes slowly and ultimately stabilized at about 0.2 . Compare the grouping $1,2,3$, the dispersion coefficient decline rate: grouping $3>$ 
grouping $2>$ grouping 1 , it takes $90 \mathrm{~min}, 60 \mathrm{~min}, 30 \mathrm{~min}$ for grouping $1,2,3$ to decreased the DO dispersion coefficient to 0.05 . So artificial flow field's improvement effect on DO distribution is obviously, and related to flow rate, the greater the flow rate, the better the improvement. Therefore, in the case of not considering energy consumption, it's better chose a pump with larger flow rate.

The Fig. 7 is the decline curve of DO dispersion coefficient of group 2, 3(the difference is nozzle shape), the figure shows that DO dispersion coefficient with circular nozzle decline more quickly than duckbill nozzle, therefore, circular nozzle is fitter than duckbill nozzle for artificial flow filed.

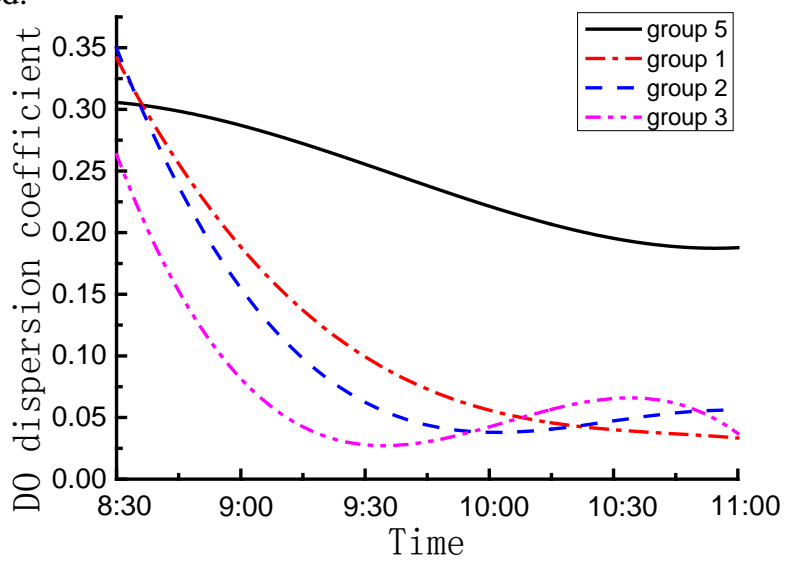

Figure 6. Dispersion trends of group 1,2,3,5

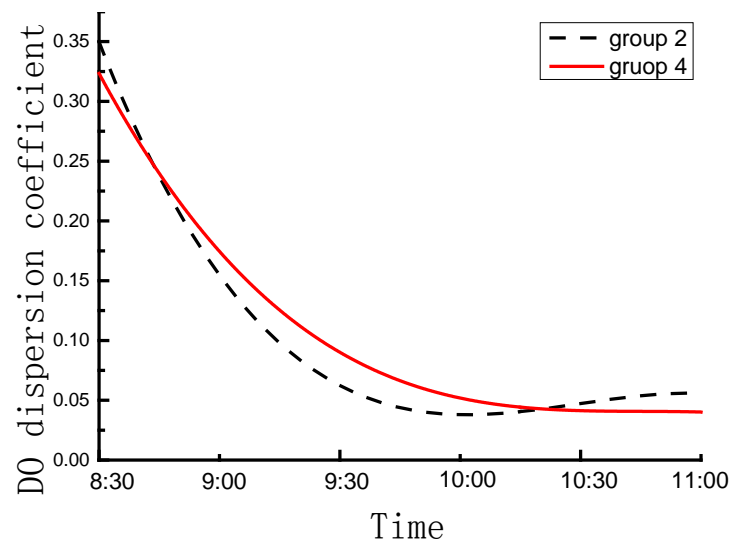

Figure 7. Dispersion trends of group 2,4

\section{SUMMARY}

We design a sets of experiment to verify the effect of artificial flow field on DO uniform distribution, RBF neural network was used to fitting the relationship of between coordinate and DO concentration to obtain the DO distribution of the whole experimental area, dispersion coefficient was used to quantify the DO distribution, the results turns to be very effective, after a period time of push flow, the terminal DO dispersion coefficient stable in about 0.05 , while the DO dispersion coefficient change slowly. Moreover, the decline rate of DO dispersion coefficient is related to flow of pumps and shape of nozzles, it's better to choose a large flow pump and circular nozzle.

\section{ACKNOWLEDGMENT}

This research was financially supported by National Nature Science Foundation of China (61563002) and Guangxi Scientific and Technological Project Fund (14251009). The corresponding author of this paper is Ai Jiaoyan, a professor at Guangxi University.

\section{REFERENCES}

[1] Dai Hengxin, Li Yingsen, Ma Xuzhou, et al. "The research of dissolved oxygen distribution in the crab ecological cultrue ponds". Journal of Shanghai Ocean University, 2013, 22(1):66-73.

[2] B.A. Cox. "A review of currently available in-stream water-quality models and their applicability for simulating dissolved oxygen in lowland rivers". Science of the Total Environment, 2003, 314316:335-77.

[3] XIEYunfeng, CHENTongbin, et al. Impact of spatial interpolation methods on the estimation of regional soil Cd. Acta Scientiae Circumstantiaen, 2010, 30(4):847-854.

[4] Guo Long, Zhang Haitao, et al. Comparison between CO-KRIGING model and geographically weighted regression model in spatial prediction of soil attributes. Acta Pedologica Sinica, 2012, 49(5):1037-1042.

[5] Li Qiquan, Wang Changquan, Yue Tianxiang, et al. "Method for spatial variety of soil organic matter based on radial basis function neural network". Transactions of the CSAE, 2010, 26(1): 87-93.

[6] Chen Feixiang, Cheng Jiachang, Hu Yueming, et al. "Spatial Prediction of Soil Properties by RBF Neural Network". Scientia Geographica Sinica, 2013, 1(1): 69-74.

[7] Liu Susu, Sun Li min. Performance comparison of regression prediction on support vector machine and RBF neural network. Computer Engineering and Design, 2011, 32(12):4202-4205.

[8] Liu Songqing. Research and application on MATLAB BP neural network. Computer Engineering and Design, 2003, 24(11):81-83.

[9] Abdi H. "Coefficient of variation”. Encyclopedia of research design, 2010: 169-171.

[10] Bendel R B, Higgins S S, Teberg J E, et al. “Comparison of skewness coefficient, coefficient of variation, and Gini coefficient as inequality measures within populations”. Oecologia, 1989, 78(3): 394-400. 